\title{
EFEITO DA ATMOSFERA MODIFICADA ATIVA NA QUALIDADE DO MELÃO 'ORANGE FLESH' MINIMAMENTE PROCESSADO
}

\author{
Effect of the active modified atmosphere in the quality of the melon \\ tipo orange flesh processed minimally
}

\author{
Francisca Marta Machado Casado de Araújo ${ }^{1}$, Antônio Vitor Machado², Adimilson Bosco Chitarra ${ }^{3}$
}

\begin{abstract}
RESUMO
O aumento da demanda por produtos minimamente processados traz um grande desafio para a ciência e tecnologia de alimentos, considerando-se a escassez de informações sobre a manutenção da qualidade desses produtos. O armazenamento destes em condições adequadas é um ponto fundamental para o sucesso dessa tecnologia. Objetivou-se com este trabalho avaliar o efeito da atmosfera modificada ativa na qualidade e conservação do melão 'Orange Flesh' minimamente processado. Os frutos, após o processamento, foram embalados sob atmosfera modificada (AM passiva - controle, AM ativa com 5\% de $\mathrm{CO}_{2}$ e $5 \%$ de $\mathrm{O}_{2}$ e AM ativa com $10 \%$ de $\mathrm{CO}_{2}$ e $2 \%$ de $\left.\mathrm{O}_{2}\right)$, armazenados em câmara fria $\left(6^{\circ} \mathrm{C} \pm 1{ }^{\circ} \mathrm{C}\right.$ e UR $\left.85 \% \pm 5 \%\right)$ durante 8 dias e as amostras retiradas para análises físicas, físico-químicas, químicas e sensoriais, a cada 2 dias de armazenamento. A atmosfera modificada ativa pouco influenciou no comportamento das variáveis $\mathrm{pH}$, acidez total titulável, firmeza, pectina total e sabor com relação à atmosfera modificada passiva. Menor solubilização de pectinas foi detectada nas amostras armazenadas sob atmosfera com $10 \%$ de $\mathrm{CO}_{2}$ e $2 \%$ de $\mathrm{O}_{2}$. Os atributos de qualidade, sabor e textura não sofreram deteriorações durante os 8 dias de armazenamento, em todos os tratamentos, de acordo com a análise sensorial.
\end{abstract}

Termos para indexação: Melão, ‘Orange Flesh', minimamente processado, atmosfera modificada, qualidade.

\section{ABSTRACT}

The increase of the demand for products processed minimally brings a great challenge for the food science and technology, being considered the shortage of information about the maintenance of the quality of those products. The storage of these products in appropriate conditions is a fundamental point for the success of this technology. The objective of this work was to evaluate the effect of the active modified atmosphere in the quality and conservation of the melon 'Orange Flesh' processed minimally. The fruits, after the processing were wrapped under modified atmosphere (passive $\mathrm{AM}$ - control; active $\mathrm{AM}$ with $5 \%$ of $\mathrm{CO}_{2}$ and $5 \%$ of $\mathrm{O}_{2}$ and activate $\mathrm{AM}$ with $10 \%$ of $\mathrm{CO}_{2}$ and $2 \%$ of $\left.\mathrm{O}_{2}\right)$, stored at $\left(6^{\circ} \mathrm{C} \pm 1{ }^{\circ} \mathrm{C}\right.$ and R.H. $\left.85 \% \pm 5 \%\right)$ during 8 days and the retired samples for analyses physics, physical-chemistries, chemistries and sensorial every 2 days of storage. The variables texture, flavor, acidity titratable and total pectin, they were not affected for the active modified atmosphere. Smaller solubilization of pectins and larger retention of neutral sugars, they were detected in melons under atmosphere with $10 \%$ of $\mathrm{CO}_{2}$ and $2 \%$ of $\mathrm{O}_{2}$. The quality attributes, flavor and texture did not suffer deteriorations during the 8 days of storage, in all the treatments, in agreement with the sensorial analysis.

Index terms: melon, processed minimally, modified atmosphere, quality.

(Recebido para publicação em 28 de julho de 2003 e aprovado em 6 de junho de 2005)

\section{INTRODUÇÃO}

A tecnologia de produtos minimamente processados (PMP) ou "fresh-cut" vem se desenvolvendo rapidamente como resposta a uma forte demanda desses produtos por parte do consumidor. $\mathrm{O}$ processamento mínimo inclui operações de seleção, lavagem, descascamento, corte, fatiamento, sanificação, centrifugação, acondicionamento e armazenamento, realizadas de modo a obter-se um produto comestível fresco e que não necessite de subseqüente preparo. Essas operações conduzem a estresses mecânicos que aceleram o metabolismo desses produtos, levando-os à rápida deterioração.
A minimização das conseqüências negativas dos ferimentos em frutas e hortaliças minimamente processadas resulta em aumento da vida de prateleira e manutenção da qualidade de sabor, aroma, aparência e valor nutricional destes produtos. Vários métodos têm sido utilizados para o controle de mudanças físicas indesejáveis que afetam a qualidade dos PMP. A refrigeração, controle de umidade, adição de químicos e as embalagens em atmosferas modificadas têm sido usados com freqüência para preservar a qualidade destes produtos e aumentar a vida de prateleira. No entanto, o conhecimento prévio das características de cada produto é essencial para o sucesso de qualquer tecnologia.

\footnotetext{
1 Professora Dra. do Departamento de Biologia/UERN - Universidade Estadual do Rio Grande do Norte.

${ }^{2}$ Mestre em Ciência dos Alimentos/UFLA - Universidade Federal de Lavras - Caixa Postal 3037 - 37.200-000 - Lavras, MG

${ }^{3}$ Professor Dr. Departamento de Ciência dos Alimentos/UFLA.
} 
O melão 'Orange Flesh', apesar de muito bem aceito pelos consumidores devido à qualidade de sua polpa, é pouco conveniente para uso individual, pois seus frutos são grandes e exigem preparo, como o descasque e a eliminação das sementes, antes do consumo. O processamento mínimo deste fruto torna-o muito prático, além de permitir um melhor aproveitamento. Com este trabalho teve se como objetivo geral avaliar o efeito da atmosfera modificada ativa na qualidade e conservação do melão 'Orange Flesh' minimamente processado, por meio de análises químicas, físicas, físico-químicas, microbiológicas e sensoriais.

\section{MATERIALEMÉTODOS}

Os melões 'Orange Flesh' utilizados foram adquiridos em estabelecimento comercial de Lavras, MG, procedentes de pomar comercial de Mossoró-RN. Os frutos selecionados em função do estádio de maturação, uniformidade de tamanho e coloração foram transportados para o Laboratório de Bioquímica de frutos do Departamento de Ciência dos Alimentos da Universidade Federal de Lavras e armazenados a $5^{\circ} \mathrm{C}$ por 24 horas, até o início do processamento.

Os melões, após lavagem com água, foram imersos em solução de cloro ativo a 200 ppm por 15 minutos, secos ao ar, descascados manualmente e fatiados em forma de leques com $5 \mathrm{~mm}$ de espessura e $30 \mathrm{~mm}$ de comprimento com o auxílio de multiprocessador MASTER AT. Em seguida, as fatias foram imersas em solução de cloro ativo a 100 ppm, por três minutos, sendo o excesso de água removido por escoamento através de peneiras especiais de plástico. Práticas de higiene foram utilizadas para a desinfecção do ambiente, facas e utensílios, com hipoclorito comercial, utilização de luvas, máscara e touca pelos manipuladores.

Os frutos processados foram acondicionados em embalagem rígida de polipropileno média barreira e selados com filme flexível de polietileno + polipropileno alta barreira, em seladora a vácuo, fazendo-se uso de injeção de gases para a obtenção de atmosfera modificada ativa (AMA $5 \% \mathrm{CO}_{2}+5 \% \mathrm{O}_{2}$ e AMA $\left.10 \% \mathrm{CO}_{2}+2 \% \mathrm{O}_{2}\right)$. Melões acondicionados nas mesmas embalagens sem a injeção de gases foram utilizados como controle (atmosfera modificada passiva-AMP). As embalagens com cerca de $190 \mathrm{~g}$ de frutos foram armazenadas em câmara fria $\left(6^{\circ} \mathrm{C} \pm\right.$ $1{ }^{\circ} \mathrm{C} \mathrm{e} \mathrm{UR} 85 \% \pm 5 \%$ ) e as amostras retiradas para análises físicas, físico-químicas, químicas e sensoriais a cada dois dias, durante um período de oito dias de armazenamento.
$\mathrm{O}$ experimento foi realizado em delineamento inteiramente casualizado (DIC), em esquema fatorial duplo ( $3 \times 5)$, com três repetições. Os fatores consistiram dos tratamentos (AMP - controle, AMA-1 com 5\% de $\mathrm{CO}_{2} \mathrm{e}$ $5 \%$ de $\mathrm{O}_{2}$ e AMA- 2 com $10 \%$ de $\mathrm{CO}_{2}$ e $2 \%$ de $\mathrm{O}_{2}$ ) e tempo de armazenamento $(0,2,4,6$ e 8 dias). Cada parcela experimental foi constituída por uma embalagem com cerca de $190 \mathrm{~g}$ de frutos. Os dados foram analisados utilizandose o programa Sistema para Análise de Variância (SISVAR), da Universidade Federal de Lavras e as médias comparadas por meio do teste de Tukey (5\%).

Foram feitas as seguintes determinações:

- $\mathrm{pH}$ - obtido do filtrado, utilizando-se potenciômetro Micronal modelo B 474, segundo técnica estabelecida pela AOAC (1992).

- Acidez total titulável (ATT) - foi obtida por titulação do filtrado com $\mathrm{NaOH} 0,1 \mathrm{~N}$, segundo a técnica estabelecida pelo Instituto Adolfo Lutz (1985) e expressa em porcentagem de ácido cítrico.

- Sólidos solúveis totais (SST) - foi determinado no filtrado por refratometria, conforme normas da AOAC (1992), utilizando-se refratômetro digital ATAGO PR-1000, e os resultados expressos em ${ }^{\circ}$ Brix.

- Açúcares solúveis totais (AST) - foram extraídos com álcool etílico a $70 \%$ e determinados, espectrofotometricamente, a $620 \mathrm{~nm}$, pelo método de antrona (DISCHE, 1962). Os resultados foram expressos como \% de glicose na polpa.

- Firmeza - foi determinada com o auxílio de analisador de textura Stable Micro System modelo TAXT2i, que mediu a força de penetração na fatia, por meio de uma sonda de aço inoxidável de $2 \mathrm{~mm}$ de diâmetro devidamente calibrada. Valores de velocidade $(5 \mathrm{~mm} / \mathrm{s})$ e distância máxima de penetração $(5 \mathrm{~mm})$ foram previamente fixados e a firmeza expressa em Newtons $(\mathrm{N})$.

Pectina total (PT) e pectina solúvel (PS) pectinas totais e solúveis foram extraídas segundo a técnica descrita por McCready \& McComb (1952), e determinadas colorimetricamente, segundo Bitter \& Muir (1962). Os resultados foram expressos em $\mathrm{mg}$ de ácido galacturônico por $100 \mathrm{~g}$ de polpa.

\section{Análise sensorial}

Os frutos minimamente processados foram avaliados por um grupo de 12 provadores treinados, selecionados de uma equipe de 20 julgadores. Para a avaliação das características de textura e sabor foi utilizada uma escala hedônica de 9 pontos, em que 9, 8, 7, 6, 5, 4, 3 ,

Ciênc. agrotec., Lavras, v. 29, n. 4, p. 817-823, jul./ago., 2005 
2 e 1 equivalem a ótima, muito boa, moderadamente boa, ligeiramente boa, indiferente, ligeiramente ruim, moderadamente ruim, muito ruim e péssima, respectivamente.

\section{RESULTADOS E DISCUSSÃO}

$\mathrm{O}$ pH das amostras sofreu influência dos tratamentos apenas no segundo e último dias de armazenamento. No segundo dia, o controle (AMP) apresentou valor de $\mathrm{pH}$ superior ao dos demais tratamentos e no oitavo dia, os valores de $\mathrm{pH}$ das amostras do controle e do tratamento AMA-1 foram superiores ao AMA-2 (Tabela 1). No presente estudo, os valores de $\mathrm{pH}$ variaram de 6,0 a 6,5, próximos aos encontrados por Lamikanra et al. (2000) que foram de 6,3 a 6,5 em melões 'Cantaloupe' minimamente processados.

Em concordância com o pH, os teores de ATT foram relativamente estáveis, com tendência à redução em todos os tratamentos. Com exceção do segundo dia de armazenamento, no qual o controle teve uma grande redução nos valores de ATT, os três tipos de atmosferas não apresentaram diferenças significativas entre si (Tabela 1). Essas oscilações, com tendência à redução nos teores de ATT, foram também observadas por Lamikanra et al. (2000). Esses autores identificaram alguns ácidos orgânicos em melões 'Cantaloupe' minimamente processados, com predominância dos ácidos cítrico e málico.

Ácidos orgânicos são importantes precursores de sabor e aroma e também, fonte de energia respiratória em células vegetais. Kays (1991) afirma que os ácidos orgânicos tendem a declinar na maioria dos frutos após a colheita e durante o armazenamento, devido à larga utilização desses compostos como substratos respiratórios e como esqueletos de carbono para a síntese de novos compostos. As respostas de vários produtos a atmosferas modificadas podem ser conflitantes. Por exemplo, o aumento de $\mathrm{CO}_{2}$ auxilia na retenção de ácidos orgânicos em tomate, mas acelera a perda de ácidos em aspargos (WILLS et al., 1998).

Nos teores de SST, houve uma diminuição no segundo dia de armazenamento para todos os tratamentos analisados, seguida de algumas oscilações, mas com valores finais reduzidos. Apenas no sexto dia de armazenamento foram observadas diferenças estatísticas entre os tratamentos, tendo o controle apresentado maiores valores de SST (Tabela 2). Portela \& Cantwell (1998) também observaram níveis estáveis de SST em melões 'Cantaloupe' minimamente processados armazenados à baixas temperaturas. O teor de sólidos solúveis é um fator tradicionalmente usado para caracterizar a qualidade do melão, ele está diretamente relacionado às condições climáticas durante a produção.

Quanto aos AST, todos os tratamentos mostraram uma elevação em seus teores até o quarto dia de armazenamento (Tabela 2). Após esta data, os valores permaneceram relativamente constantes para os melões armazenados sob atmosfera modificada ativa, enquanto que no controle houve uma diminuição permanente destes, até o final do armazenamento, provavelmente devido ao consumo de substratos durante o metabolismo respiratório dos frutos.

TABELA 1 - Valores médios de $\mathrm{pH}$ e acidez total titulável (ATT) do melão 'Orange Flesh' minimamente processado, armazenado a $6 \pm 1^{\circ} \mathrm{C}$ e $85 \% \pm 5 \%$ de UR, durante 8 dias (controle $-\mathrm{AMP}, 5 \% \mathrm{CO}_{2}$ e $5 \% \mathrm{O}_{2}-\mathrm{AMA}-1$, $10 \% \mathrm{CO}_{2}$ e $2 \% \mathrm{O}_{2}$ AMA-2).

\begin{tabular}{lccccc}
\hline \multirow{2}{*}{ Tratamentos } & \multicolumn{5}{c}{ Armazenamento (dias) } \\
\cline { 2 - 6 } & $\mathbf{0}$ & $\mathbf{2}$ & $\mathbf{4}$ & $\mathbf{6}$ & $\mathbf{8}$ \\
AMP & $6,07 \mathrm{a}$ & $6,43 \mathrm{a}$ & $6,23 \mathrm{a}$ & $6,13 \mathrm{a}$ & $6,47 \mathrm{a}$ \\
AMA-1 & $6,07 \mathrm{a}$ & $6,13 \mathrm{~b}$ & $6,27 \mathrm{a}$ & $6,13 \mathrm{a}$ & $6,30 \mathrm{a}$ \\
AMA-2 & $6,00 \mathrm{a}$ & $6,13 \mathrm{~b}$ & $6,10 \mathrm{a}$ & $6,10 \mathrm{a}$ & $6,03 \mathrm{~b}$ \\
& & ATT $(\%$ ácido cítrico) & & \\
AMP & $0,096 \mathrm{a}$ & $0,043 \mathrm{~b}$ & $0,055 \mathrm{a}$ & $0,068 \mathrm{a}$ & $0,064 \mathrm{a}$ \\
AMA-1 & $0,092 \mathrm{a}$ & $0,077 \mathrm{a}$ & $0,055 \mathrm{a}$ & $0,072 \mathrm{a}$ & $0,068 \mathrm{a}$ \\
AMA-2 & $0,094 \mathrm{a}$ & $0,072 \mathrm{a}$ & $0,052 \mathrm{a}$ & $0,068 \mathrm{a}$ & $0,068 \mathrm{a}$ \\
\hline
\end{tabular}

Médias seguidas por letras distintas, na coluna, diferem entre si pelo teste de Tukey $(\mathrm{p} \leq 0,05)$. 
TABELA 2 - Valores médios de sólidos solúveis totais (SST) e açúcares solúveis totais (AST) do melão 'Orange Flesh' minimamente processado, armazenado a $6 \pm 1{ }^{\circ} \mathrm{C}$ e $85 \% \pm 5 \%$ de UR, durante 8 dias (controle - AMP, $5 \%$ $\mathrm{CO}_{2}$ e $5 \% \mathrm{O}_{2}-\mathrm{AMA}-1,10 \% \mathrm{CO}_{2}$ e $2 \% \mathrm{O}_{2}$ AMA-2).

\begin{tabular}{lccccc}
\hline \multirow{2}{*}{ Tratamentos } & \multicolumn{5}{c}{ Armazenamento (dias) } \\
\cline { 2 - 6 } & $\mathbf{0}$ & $\mathbf{2}$ & $\mathbf{4}$ & $\mathbf{6}$ & $\mathbf{8}$ \\
AMP & $9,2 \mathrm{a}$ & $9,1 \mathrm{a}$ & $8,9 \mathrm{a}$ & $9,6 \mathrm{a}$ & $9,0 \mathrm{a}$ \\
AMA-1 & $9,5 \mathrm{a}$ & $8,5 \mathrm{a}$ & $8,9 \mathrm{a}$ & $8,4 \mathrm{~b}$ & $8,4 \mathrm{a}$ \\
AMA-2 & $9,2 \mathrm{a}$ & $8,5 \mathrm{a}$ & $8,4 \mathrm{a}$ & $8,1 \mathrm{~b}$ & $8,6 \mathrm{a}$ \\
& & AST (g/ 100) polpa) & & \\
AMP & $7,37 \mathrm{a}$ & $7,53 \mathrm{a}$ & $8,74 \mathrm{a}$ & $8,68 \mathrm{a}$ & $7,57 \mathrm{a}$ \\
AMA-1 & $6,88 \mathrm{~b}$ & $7,12 \mathrm{~b}$ & $7,62 \mathrm{~b}$ & $7,39 \mathrm{~b}$ & $7,63 \mathrm{a}$ \\
AMA-2 & $6,74 \mathrm{~b}$ & $7,12 \mathrm{~b}$ & $7,81 \mathrm{~b}$ & $7,69 \mathrm{~b}$ & $7,82 \mathrm{a}$ \\
\hline
\end{tabular}

Médias seguidas por letras distintas, na coluna, diferem entre si pelo teste de Tukey ( $\mathrm{p} \leq 0,05)$.

A respiração pode ser descrita como a quebra oxidativa dos materiais mais complexos presentes normalmente em células, tais como: amido, ácidos orgânicos e açúcares, em moléculas mais simples, tais como dióxido de carbono e água, com a conseqüente produção de energia e outras moléculas que podem ser usadas pela célula para reações sintéticas (WILLS et al., 1998). Níveis de $\mathrm{CO}_{2}$ entre $5 \%$ e $20 \%$ reduzem efetivamente a taxa respiratória na maioria dos produtos hortícolas. A inibição da respiração pode ser resultante de alterações na rota glicolítica, no metabolismo fermentativo, no ciclo de Krebs ou no sistema de transporte de elétrons, via efeito do $\mathrm{CO}_{2}$ sobre a síntese, degradação, inativação ou inibição de algumas enzimas que compõem essas rotas metabólicas.

Os valores de firmeza no melão minimamente processado diminuíram no decorrer do armazenamento em todos os tratamentos (Tabela 3). Embora não tenha havido diferenças significativas entre os tratamentos a partir do quarto dia de armazenamento, uma menor perda de firmeza foi observada para as fatias do tratamento AMA-2 $(5,4 \%)$ quando comparada ao controle (20,9\%) e AMA-1 $(21,2 \%)$, no final do armazenamento. Melões 'Honeydew' minimamente processados também reduziram a perda de firmeza quando armazenados a $5^{\circ} \mathrm{C} \mathrm{em}$ atmosfera com $15 \%$ $\mathrm{CO}_{2}$ (PORTELA \& CANTWELL, 1998).

As amostras do tratamento AMA-2 apresentaram, em geral, maiores valores de PT quando comparadas àquelas dos outros tratamentos. No entanto, diferenças significativas foram observadas apenas nos tempos 2 e 4 dias de armazenamento (Tabela 3). Menezes (1996), trabalhando com melões, também não observou diferenças significativas nos níveis de pectina total durante a maturação.

A solubilização de pectinas aumentou a partir do segundo dia de armazenamento para todos os tratamentos analisados (Tabela 3). Atmosferas modificadas ativas proporcionaram menor solubilização de substâncias pécticas, indicando tais tratamentos como mais efetivos neste caso. $\mathrm{O}$ aumento na solubilização das substâncias pécticas se associou perfeitamente com a redução da textura observada durante o armazenamento dos frutos à semelhança de outros autores (TEIXEIRA et al., 2001).

Atmosferas controladas retardam o amadurecimento e amaciamento dos frutos. Elevada concentração de $\mathrm{CO}_{2}$ proporciona maiores efeitos na retenção de firmeza que concentrações de $\mathrm{O}_{2}$ reduzidas (KADER, 1986), talvez, por sua maior influência na redução da atividade das enzimas pécticas. Lima et al. (2000), estudando a perda de firmeza em maçãs, observaram que frutos armazenados sob atmosferas com $3 \mathrm{kPa} \mathrm{CO}+1 \mathrm{kPa} \mathrm{O}_{2}$ apresentaram, em média, menor atividade de pectinametilesterase (PME) que os submetidos à atmosferas com $1 \mathrm{kPa} \mathrm{CO}_{2}+1 \mathrm{kPaO}_{2}$, demonstrando que esta enzima não foi inibida pelo baixo teor $\mathrm{de}_{2}$, mas somente pelo alto de $\mathrm{CO}_{2}$. 


\section{Análise sensorial}

De acordo com a Tabela 4, é possível observar que as notas para a variável sabor permaneceram na média de 7,4, correspondendo a valores entre "moderadamente boa" e "muito boa". Diferenças significativas ocorreram apenas nos tempos 4 e 6, em que o controle recebeu maiores notas, diferenciando-se dos outros tratamentos. Estes resultados podem ser relacionados aos maiores teores de açúcares solúveis totais apresentados nas fatias do controle, nestes dois tempos. Já no oitavo dia, os frutos com AMA-1 e AMA-2 se sobressaíram, tendo uma melhor aceitação entre os provadores.

Não foi detectado pelos provadores sabor não característico, que poderia ter se desenvolvido devido à falta de $\mathrm{O}_{2}$ e/ou excesso de $\mathrm{CO}_{2}$ no interior da embalagem.
A textura nos melões minimamente processados não foi afetada pelos tratamentos ao longo do armazenamento (Tabela 4). No entanto, os frutos armazenados em AMP apresentaram melhores notas até o sexto dia, sendo superados pelos tratamentos em atmosfera modificada ativa no final do armazenamento. As notas para esta variável corresponderam também aos valores relativos a "moderadamente boa" e "muito boa".

Embalagens sob atmosfera modificada têm sido descritas como um método que reduz a perda de textura em frutas minimamente processadas durante o armazenamento. Características sensoriais em melões rendilhados minimamente processados armazenados sob atmosfera modificada com $7 \% \mathrm{CO}_{2}$ e $12 \% \mathrm{O}_{2}$ foram mantidas durante 9 dias a $3^{\circ} \mathrm{C}$ (ARRUDA, 2002).

TABELA 3 - Valores médios de textura, pectina total (PT) e pectina solúvel (PS) do melão 'Orange Flesh' minimamente processado, armazenado a $6 \pm 1{ }^{\circ} \mathrm{C}$ e $85 \% \pm 5 \%$ de $\mathrm{UR}$, durante 8 dias (controle $-\mathrm{AMP}, 5 \% \mathrm{CO}$ e $5 \%$ $\mathrm{O}_{2}$ - AMA-1, $10 \% \mathrm{CO}_{2}$ e $2 \% \mathrm{O}_{2}$ AMA-2).

\begin{tabular}{lccccc}
\hline \multirow{2}{*}{ Tratamentos } & \multicolumn{5}{c}{ Armazenamento (dias) } \\
\cline { 2 - 6 } & 0 & 2 & 4 & 6 & 8 \\
AMP & $2,77 \mathrm{a}$ & $2,29 \mathrm{~b}$ & $2,13 \mathrm{a}$ & $2,12 \mathrm{a}$ & $2,19 \mathrm{a}$ \\
AMA-1 & $2,64 \mathrm{ab}$ & $3,01 \mathrm{a}$ & $2,34 \mathrm{a}$ & $2,04 \mathrm{a}$ & $2,08 \mathrm{a}$ \\
AMA-2 & $2,24 \mathrm{~b}$ & $2,18 \mathrm{~b}$ & $2,20 \mathrm{a}$ & $2,14 \mathrm{a}$ & $2,09 \mathrm{a}$ \\
& & PT (mg ácido galacturônico/100 g polpa) \\
AMP & $279,32 \mathrm{a}$ & $272,35 \mathrm{ab}$ & $283,23 \mathrm{a}$ & $286,62 \mathrm{a}$ & $280,15 \mathrm{a}$ \\
AMA-1 & $276,22 \mathrm{a}$ & $263,75 \mathrm{~b}$ & $258,17 \mathrm{~b}$ & $274,27 \mathrm{a}$ & $286,07 \mathrm{a}$ \\
AMA-2 & $282,33 \mathrm{a}$ & $284,01 \mathrm{a}$ & $287,84 \mathrm{a}$ & $279,52 \mathrm{a}$ & $287,08 \mathrm{a}$ \\
& \multicolumn{5}{c}{$\mathrm{PS}(\mathrm{mg}$ ácido galacturônico/100 g polpa) } \\
AMP & $35,92 \mathrm{~b}$ & $39,68 \mathrm{ab}$ & $45,10 \mathrm{~b}$ & $50,05 \mathrm{a}$ & $51,64 \mathrm{a}$ \\
AMA-1 & $35,89 \mathrm{~b}$ & $37,54 \mathrm{~b}$ & $36,16 \mathrm{c}$ & $36,50 \mathrm{~b}$ & $37,65 \mathrm{~b}$ \\
AMA-2 & $48,35 \mathrm{a}$ & $40,74 \mathrm{a}$ & $49,87 \mathrm{a}$ & $49,73 \mathrm{a}$ & $50,32 \mathrm{a}$ \\
\hline
\end{tabular}

Médias seguidas por letras distintas, na coluna, diferem entre si pelo teste de Tukey $(\mathrm{p} \leq 0,05)$. 
TABELA 4 - Valores médios de sabor e textura do melão 'Orange Flesh' minimamente processado, armazenado a $6 \pm$ $1^{\circ} \mathrm{C}$ e $85 \% \pm 5 \%$ de UR, durante 8 dias (controle - AMP, 5\% $\mathrm{CO}_{2}$ e $5 \% \mathrm{O}_{2}-\mathrm{AMA}-1,10 \% \mathrm{CO}_{2}$ e $2 \% \mathrm{O}_{2} \mathrm{AMA}-2$ ).

\begin{tabular}{lccccc}
\hline \multirow{2}{*}{ Tratamentos } & $\mathbf{5}$ Armazenamento (dias) \\
\cline { 2 - 5 } & $\mathbf{0}$ & $\mathbf{2}$ & $\mathbf{4}$ & $\mathbf{6}$ & $\mathbf{8}$ \\
AMP & $7,5 \mathrm{a}$ & $7,5 \mathrm{a}$ & $7,6 \mathrm{a}$ & $7,4 \mathrm{a}$ & $7,4 \mathrm{a}$ \\
AMA-1 & $7,4 \mathrm{a}$ & $7,5 \mathrm{a}$ & $6,9 \mathrm{~b}$ & $7,2 \mathrm{ab}$ & $7,5 \mathrm{a}$ \\
AMA-2 & $7,1 \mathrm{a}$ & $7,8 \mathrm{a}$ & $7,5 \mathrm{ab}$ & $6,7 \mathrm{~b}$ & $7,8 \mathrm{a}$ \\
\multicolumn{7}{c}{ Textura (notas) } \\
AMP & $7,5 \mathrm{a}$ & $7,5 \mathrm{a}$ & $7,7 \mathrm{a}$ & $7,7 \mathrm{a}$ & $7,6 \mathrm{a}$ \\
AMA-1 & $7,0 \mathrm{a}$ & $7,2 \mathrm{a}$ & $7,2 \mathrm{a}$ & $7,7 \mathrm{a}$ & $8,0 \mathrm{a}$ \\
AMA-2 & $7,1 \mathrm{a}$ & $7,7 \mathrm{a}$ & $7,5 \mathrm{a}$ & $7,2 \mathrm{~b}$ & $7,8 \mathrm{a}$ \\
\hline
\end{tabular}

Médias seguidas por letras distintas, na coluna, diferem entre si pelo teste de Tukey, $(\mathrm{p} \leq 0,05)$.

\section{CONCLUSÕES}

A atmosfera modificada ativa pouco influenciou no comportamento das variáveis $\mathrm{pH}$, acidez total titulável, firmeza, pectina total e sabor, quando comparada à atmosfera modificada passiva. Mas, embora a firmeza não tenha sido influenciada pela atmosfera modificada ativa, as fatias armazenadas em $10 \% \mathrm{CO}_{2}$ e $2 \% \mathrm{O}_{2}$ apresentaram menor solubilização de pectinas.

Atmosferas modificadas (passiva e ativa) foram eficientes na manutenção dos atributos de qualidade, sabor e textura, de melões 'Orange Flesh' minimamente processados, durante os 8 dias de armazenamento previstos, como demonstrado pela análise sensorial.

\section{REFERÊNCIAS BIBLIOGRÁFICAS}

ARRUDA, M. C. Processamento mínimo de melão rendilhado: tipo de corte, temperatura de armazenamento e atmosfera modificada. 2002. 71 f. Dissertação (Mestrado em Ciências dos Alimentos) - Escola Superior de Agricultura Luiz de Queiroz, Piracicaba, 2002.

ASSOCIATION OF OFFICIAL AGRICULTURAL CHEMISTS. Official methods of the Association of the Official Agricultural Chemists. 12.ed. Washington, 1992.1015p.

BITTER, T.; MUIR, H. M. A modified uronic acid carbazole reaction. Analytical Biochemistry, New York, v. 34, n. 4, p. 330-334, Feb. 1962.
DISCHE, E. Color reactions of carbohydrates. In: WHISTLER, R. L.; WOLFRAM, M. L. (Eds.). Methods in carbohydrates chemistry. New York: Academic, 1962. v. 1, p. 477-512.

INSTITUTO ADOLFO LUTZ. Normas analíticas, métodos químicos e físicos para análise de alimentos. 3. ed. São Paulo, 1985. v. 1, 533 p.

KADER, A. A. Biochemical and physiological basis for effects of controlled and modified atmospheres on fruits and vegetables. Food Technology, Chicago, v. 40, n. 5, p. 99-104, May 1986.

KAYS, J. S. Postharvest physiology of perishables plant products. New York: AVI, 1991.534 p.

LAMIKANRA, O.; CHEN, J. C.; BANKS, D.; HUNTER, P. A. Biochemical and microbial changes during the storage of minimally processed cantaloupe. Journal of Agricultural and Food Chemistry, Washington, v. 48, n. 12, p. 5955-5961, Dec. 2000.

LIMA, L. C.; BRACKMANN, A.; CHITARRA, M. I. F.; MORAES, A. R.; COSTA, J. R. Perda de firmeza da polpa de maçãs (Malus domestica, Borkh) "Royal Gala" armazenadas sob refrigeração e atmosfera controlada. Revista Brasileira de Fruticultura, Jaboticabal, v. 22, p. 26-31, jul. 2000. Número Especial. 
McCREADY, P. M.; McCOMB, E. A. Extration and determination of total pectic material. Analytical Chemistry, Washington, v. 24, n. 12, p. 1586-1588, Dec. 1952.

MENEZES, J. B. Qualidade pós-colheita de melão tipo galia durante a maturação e o armazenamento. 1996. 157 f. Tese (Doutorado em Ciência dos Alimentos) - Universidade Federal de Lavras, Lavras, 1996.

PORTELA, S. I.; CANTWELL, M. I. Quality changes of minimally processed honeydew melons stored in air or controlled atmosphere. Postharvest Biology and Technology, California, v. 14, p. 351-357, 1998.

TEIXEIRA, G. H.A.; DURIGAN, J. F.; MATTIUZ, B.; ROSSI JÚNIOR, O. D. Processamento mínimo de mamão 'Formosa'. Ciência e tecnologia de alimentos, Campinas, v. 21, n. 1, p. 47-50, jan./abr. 2001.

WILLS, R.; McGLASSON, B.; GRAHAM, D.; JOYCE, D. Postharvest: an introduction to the physiology \& handling of fruit, vegetables \& ornamentals. Austrália: [s.n.], 1998. $262 \mathrm{p}$. 